\title{
Severe jejunitis masquerading as intussusception in a case of Henoch-Schönlein purpura
}

\author{
B Mehra, (iD) V Aggarwal, (iD) H Wardhan, (iD) SK Dugaya iD \\ Paediatric Intensive Care Unit, Max Superspeciality Hospital, India
}

Corresponding author, email: bharatmehra909@gmail.com

\section{Summary}

Intussusception is a well-known complication of Henoch-Schönlein purpura (HSP). ${ }^{1}$ Diagnosis of intussusception is based on well-defined clinical and radiological features, but certain conditions can mimic intussusception, both clinically and radiologically, so closely as to result in unnecessary invasive procedures. Authors here present a case of HSP complicated by severe acute jejunitis, masquerading as jejuno-jejunal intussusception on ultrasound.

\section{Case report}

A 9-year-old female child presented with worsening colicky abdominal pain associated with non-bilious vomiting for five days and passing semi-formed stools for two days. There was no history of fever, blood in stools, trauma, joint pains, icterus, haematuria or decreased urine output. Systemic examination revealed a slightly distended but non tender abdomen with no organomegaly and normal bowel sounds.

An abdominal radiograph revealed a distended transverse and descending colon, without any significant air fluid levels. Initial workup comprising a blood count, liver and renal function tests, and serum lipase were within normal limits except for an elevated C-reactive protein (CRP) level. She was managed conservatively with intravenous fluids, antibiotics and analgesia. Over the next 24 hours, she developed a discrete maculopapular rash over her hands and feet, which became progressively more prominent and evolved into purpuric spots and blotches (Figure 1B and C). Tests for coagulation were normal. Her urine examination revealed evidence of nephropathy in the form of significant proteinuria (spot protein-creatinine ratio 0.58). A clinical diagnosis of HSP was made and dexamethasone was started $(0.15 \mathrm{mg} / \mathrm{kg} /$ day in four divided doses $)$. Despite this therapy, her abdominal pain worsened, and she developed bilious vomiting with fresh rectal bleeding. Ultrasound abdomen showed a long segment jejuno-jejunal intussusception(Figure 1A). After a paediatric surgical consultation, she was taken for exploratory laparotomy and to the surgeon's surprise, intraoperative findings revealed thick, inflamed, dusky, friable jejunal loops with foci of sub-serosal haemorrhages (Figure 1D) without any evidence of intussusception. Around 30 inches of jejunum was found to be severely inflamed and had to be resected. Postoperatively, the child had rectal bleeding for a couple of days. As few cases of acute jejunitis have been reported in association with HSP, a diagnosis of HSP complicated with acute jejunitis was kept.
Steroids were reinitiated from the fourth postoperative day (delayed to avoid potential anastomotic leak). Subsequently, she showed remarkable clinical improvement with complete resolution of purpuric rash and rectal bleeding within three days of re-starting dexamethasone.

Microscopic examination of the resected bowel segment revealed focal denudation of epithelium with haemorrhages, foci of cryptitis, dense perivascular neutrophilic infiltrates, focal fibrinoid necrosis along with serositis and was reported to be consistent with diagnosis of leukocytoclastic vasculitis. In view of the persistent proteinuria, a renal biopsy was done and revealed a focal endocapillary proliferative IgA nephropathy, consistent with the diagnosis of HSP. Steroid dosage was successfully tapered off after two weeks without any recurrence of rectal bleeding, rashes, puffiness or abdominal pain. She is currently being managed as an outpatient for persistent proteinuria.

\section{Discussion}

Intussusception is diagnosed based on clinical features (triad of intermittent abdominal pain, vomiting, and bloody mucoid stools) and classical radiological features seen on ultrasonography (USG) - target sign on transverse scan and a pseudo-kidney sign on longitudinal scan., ${ }^{2,3}$ Intussusception occurring during infancy usually does not have a lead point. However, in children over five years of age, a pathologic lead point can be found in the majority of cases. ${ }^{4}$ Meckel's diverticulum and intestinal polyp are the most common lead points, ${ }^{4}$ but there are a myriad of other rare causes that include HSP and polyarteritis nodosa.

Certain conditions with radiological features similar to intussusceptions can pose a diagnostic dilemma for the clinicians. In their series of seven cases of intussusception mimickers, Karakus et al. reported three children with underlying HSP with features suggestive of intussusceptions. The subsequent complete resolution of USG abnormality in them, without any active intervention, was attributed to intramural haemorrhage mimicking as intussusception. ${ }^{3}$ 

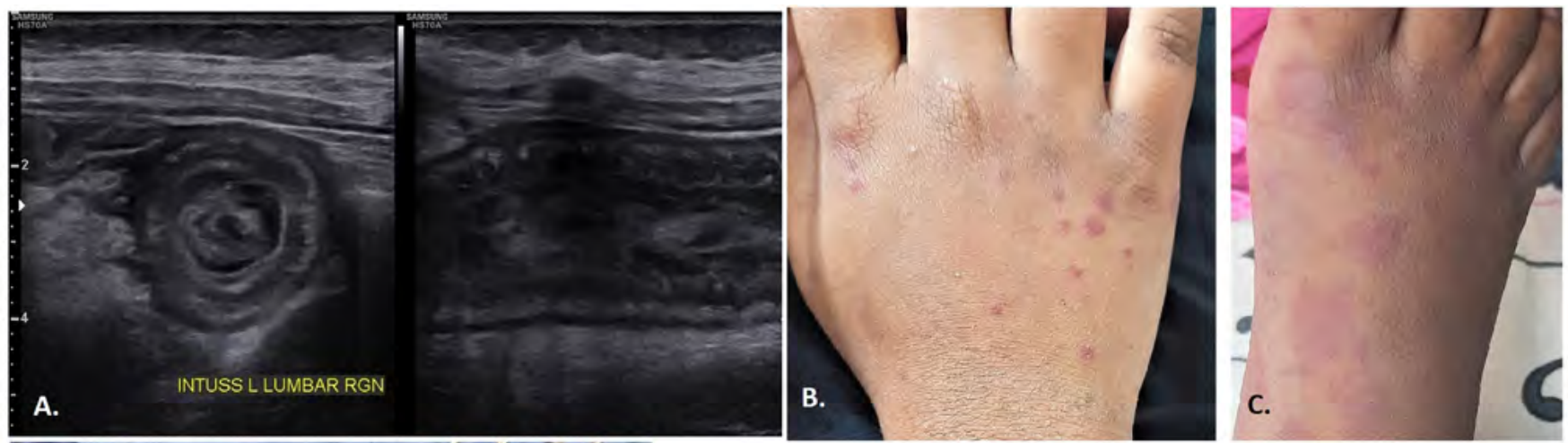

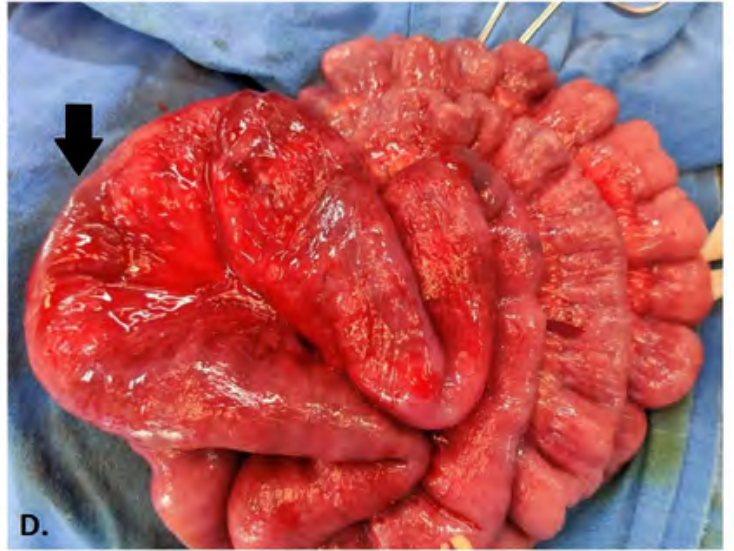

In our case, severe clinical symptoms in association with USG and evidence of jejuno-jejunal intussusception necessitated surgical exploration. However, instead of intussusception, acute severe jejunitis was found to be the mimicker of intussusception, clinically as well as radiologically.

Acute obstructive jejunitis has been reported as a rare feature of HSP. ${ }^{4}$ Gunasekharan et al. reported four children with duodenojejunal inflammation, in whom the typical rash of HSP was either not present or appeared late. ${ }^{5}$ Similarly, Chesler et al. reported a case of severe jejunitis presenting with abdominal pain and melaena, with atypical skin lesions in which the diagnosis of HSP was delayed until the appearance of classical skin manifestations. ${ }^{6}$ In their case, symptoms resolved after commencement of steroids. Similarly, in our case, classical skin lesions appeared on day two of admission (day seven of illness), by then she already had features of acute abdominal emergency. The authors feel that early recognition of the aetiology and initiation of steroids could have led to resolution of gastrointestinal (GI) symptoms and avoided surgery.

As illustrated by our case, severe jejunitis, which is a known accompaniment of HSP, can mimic intussusception or can be the source of a transient intussusception and therefore should be kept in mind as a differential diagnosis when exploration is undertaken.

\section{Conflict of interest}

The authors declare no conflict of interest.

\section{Funding source}

None.

\section{ORCID}

B Mehra (D) https://orcid.org/0000-0001-8194-7623 V Aggarwal (iD https://orcid.org/0000-0003-4767-0346 H Wardhan (iD https://orcid.org/0000-0002-5152-9735 SK Dugaya (iD https://orcid.org/0000-0003-2154-6144

\section{REFERENCES}

1. Choong CK, Beasley SW. Intra-abdominal manifestations of Henoch-Schönlein purpura. J Paediatr Child Health. 1998;34(5):405-9. https://doi.org/10.1046/j.1440-1754.1998. 00263.x. PMID: 9767498.

2. Stringer MD, Pablot SM, Brereton RJ. Paediatric intussusception. Br J Surg. 1992;79:867-76. https://doi.org/10. 1002/bjs.1800790906. PMID: 8581242.

3. Karakus SC, Ozokutan BH, Ceyla H. Diseases mimicking intussusception: Diagnostic dilemma. Pediatr Int. 2014;56:7 68-71. https://doi.org/10.1111/ped.12334.

4. Balf CL. The alimentary lesion in anaphylactoid purpura. Arch Dis Child. 1951;26(125):20-7. https://doi.org/10.1136/ adc.26.125.20. PMID: 14811523 .

5. Gunasekharan TS, Berman J, Gonzalez M. Duodenojejunitis: is it idiopathic or is it Henoch-Schönlein purpura without the purpura? J Ped Gastroenterol Nutr. 2000;30(1):22-8. https://doi.org/10.1097/00005176-200001000-00013. PMID: 10630435.

6. Chesler L, Hwang L, Wendy P, Heyman MB. HenochSchönlein purpura with severe jejunitis and minimal skin lesions. J Ped Gastroenterol Nutr. 2000;30(1):92-5. https://doi. org/10.1097/00005176-200001000-00024. PMID: 10630446. 